Pre-print: Hunady (2019). The effect of the Internet on corruption awareness and corruption incidence in the EU. In Information Polity, Vol. 24, No.1, pp. 75-89. DOI: https://doi.org/10.3233/IP-180086

https://content.iospress.com/articles/information-polity/ip180086

\title{
The effect of the Internet on corruption awareness and corruption
}

\section{incidence in the $\mathbf{E U}$}

\begin{abstract}
It is obvious that the Internet connection brings several unquestionable benefits to people and society. However, not all of them can be seen at first sight. This study examines the potential effect of Internet usage on corruption awareness as well as experience with corruption and actual reporting of corruption cases. We conducted mainly IV probit and 2SLS regression based on the data from the Eurobarometer survey. We have found that intensity of Internet usage seems to be a significant factor affecting respondents' knowledge about where to report the corruption. Internet usage appears to have a positive effect on the accuracy of corruption extent estimation in a country. Moreover, our results strongly suggest that those using the Internet frequently are more likely to report experiences of corruption. In line with this result, promoting of Internet usage and enabling Internet access can be seen as a potential anti-corruption tool. Our findings have several important implications for anti-corruption and bribery policies. Due to the fact that especially the initial installation cost proves to be the essential problem, the effective strategy should also include the subsidies for Internet access.
\end{abstract}

\section{Key points for practitioners:}

- Respondents who are using the Internet more often have a better awareness of corruption, and they are more often willing to report the corruption.

- The public support of internet usage seems to be a potentially effective anti-corruption policy,

- Especially relatively high initial installation cost seems to be the most significant barrier to Internet adoption and usage.

\section{Keywords}

Corruption awareness, Reporting corruption, Determinants of corruption, Internet, Internet usage, Anti-corruption policy. 


\section{Introduction}

Despite the fact that the corruption is often associated with less developed countries, this problem is still significant in almost every country in the world. The effect of corruption on economies and society in general is considered as strongly negative. As a result of this fact, causes of corruption have been extensively examined in the economic literature in recent years. Several determinants of corruption have been identified by empirical studies at micro and macro level so far. With respect to the socioeconomic factors, education, income and marital status appear to be significant determinants of corruption involvement (Mocan 2008). Furthermore, most of the studies conclude that, in general, women are less corrupt than man (Rivas 2012; Torgler \& Valev 2010). From a macroeconomic point of view, inflation, economic development, economic freedom, income distribution (Ata \& Arvas, 2011) as well as economic uncertainty (Goel and Ram, 2013) seem to be significantly related to corruption. Moreover, Rehman \& Naveed (2007) found that GDP per capita, the unemployment rate, public expenditure and secondary school enrolments are significant factors influencing the level of corruption in a country. The essential role of the quality of institutions in preventing corruption has been highlighted in several studies (Hunady, 2017; Dreher et al., 2009; Mocan, 2008; Sööt \& Rootalu, 2012). The rule of law is often reported as the crucial variable related to institutions and their effect on corruption (Elbahnasawy \& Revier, 2012; Kim, 2014; Iwasaki and Suzuki, 2012). With respect to this issue Andrés \& Goel (2011) argue that the higher levels of corruption are also correlated with higher levels of software piracy in the country.

Serra (2006) states that corruption is significantly less present in richer countries with democratic institutions and mainly protestant population. Regarding the effect of democracy, Kalenborn \& Lessmann (2013) argue that democracy and press freedom are two of the most important factors in controlling the corruption in the country. Similar results have been obtained by Bhattacharyya \& Hodler (2015). Using panel data covering 129 countries in the period between 1980 and 2007, the authors provide empirical support for the assumption that media freedom and democratisation jointly reduce the level of political corruption in the country. A similar effect of press freedom has been also found by Brunetti \&Weder (2003). 
Based on these results it seems very likely that the role of unbiased information is an important aspect in reducing corruption. This is, to a large extent, also confirmed by DiRienzo et al. (2007) who found that better access to information via information and communication technologies could significantly decrease the extent of corruption in a country. Today the Internet can be considered as the most powerful source of information. As reported by Orviska \& Hudson (2009), Internet technology has the potential to reduce past disadvantages of other source of information such as lack of access to information by those living in remote areas. In line with this, we believe that the Internet could be an important piece of the puzzle in fighting the corruption in all regions. As stated by Andersen, Bentzen, Dalgaard \& Selaya (2011), the Internet could reduce corruption in several ways. First of all, the Internet as the major source of information may increase the probability of corruption detection. Second, the Internet is the main vehicle for the provision of e-government, something that allows citizens access to government services online, thus limiting the interaction between potentially corrupt officials and the public. Moreover, online systems require standardized rules and procedures and this could reduce bureaucratic discretion and increase transparency. The potential anti-corruption effect of Internet could be explained by better access to information and higher transparency. This could further lead to high corruption awareness as well as more accurate knowledge about where to report corruption. However, so far, in the theoretical and empirical economic literature, little attention has been paid to effect of the Internet on corruption although there are some studies focused on egovernment or testing the effect of the Internet on corruption based on cross-sectional macro-level data.

Andersen (2009) has focused attention on the potential anti-corruption effect of e-government. Using data for 149 countries for two periods of time he found significant and economically interesting effects of e-government introduction. His results suggest that, by the most conservative estimate, moving from the $10^{\text {th }}$ percentile to the $90^{\text {th }}$ percentile in the e-government ranking distribution implies a reduction in corruption equivalent to moving from the $10^{\text {th }}$ to $23^{\text {rd }}$ percentile in the control of corruption distribution. Shim \& Eon (2008) as well as Elbahnasawy (2014) both found that egovernment is an effective tool in fighting corruption. The effect is significantly strengthened by greater Internet adoption in a country. Similar positive effect of e-government on reducing corruption 
has recently been further confirmed by the results of several other studies (Choi, 2014; Kim, 2014, 2015; Ionescu, 2015; Zhao $\& \mathrm{Xu}, 2015)$. Internet adoption and technical skills are mostly seen as complementary to the successful adoption of e-government as effective anti-corruption strategy.

Other research supports these conclusions. Garcia-Murillo (2010) based on the cross-section data for approximately 170 countries identified a positive impact of Internet access on reducing corruption. Goel et al. (2012) assumed that greater corruption awareness could reduce corruption and the Internet was considered the main source of information about the corruption. The authors analysed data from Google and Yahoo and found that Internet hits about corruption and bribery per capita correlate negatively with corruption perceptions as well as with the incidence of corruption in a given country. However, the effect of the Internet may not be exclusively positive. For example, Im et al. (2014) found that increased time spent on the Internet could lower citizens' degree of trust in government as well as their level of compliance with regulations. Another potentially useful tool with which to fight corruption, which is enabled by the Internet, is crowdsourcing. New technologies which involve more people in policy processes could be beneficial to inter alia, anti-corruption policies. The potential benefits of crowdsourcing for government and public policy have been summarised by Lehdonvirta \& Bright (2015) and the use of crowdsourcing for corruption reporting has been discussed in detail by Zinnbauer (2015). Collective actions and social mobilisation against corruption in this dimension have become possible only in recent years thanks to the emergence of new technologies.

The short-run and long-run effect of Internet usage on corruption have been analysed by Hunady \& Orviska (2015) using the panel cointegration approach and vector-error correction models. The panel data include 86 countries over the period 1998-2012. The results strongly support the existence of short-run as well as a stable long-run relationship between both variables. According to the results, the share of Internet users in the population is negatively correlated with corruption in the short-run as well as in the long run.

This paper aims to test the assumed effect of Internet access as well as Internet usage on corruption related variables based on the micro-level data from the Eurobarometer survey. The paper is mostly empirically oriented, using the results of regression analysis. We examine the effect of Internet usage on corruption awareness, experience with corruption and its reporting. In the next 
section we describe the methodology and data used in this analysis. Subsequently, we present and discuss the results of the analysis followed by conclusions and important policy implications of our findings.

\section{Data and methodology}

We used regression models in order to test the potential relationships between Internet usage and corruption related variables. In particular, probit regressions have been used, using the binary dependent variables. Due to potential problems with erogeneity we applied also used probit with Newey's (1987) two step estimator using instrumental variable (IV probit) as well as two stage leastsquares (2SLS) regression analysis. This methodology allows us to correct for endogeneity bias. Independent variables have been tested for endogeneity by using Wald tests of the exogeneity (for IV probit) and Durbin-Wu-Hausman test (for 2SLS). In the case of suspected endogeneity, we take into the account the results of the two-stage regressions. We use both two-stage as well as standard probit estimates in order to check the robustness of our results. Independent variables are capturing frequency of Internet usage at home as well as daily or weekly usage of the Internet anywhere and examine its potential effect on corruption awareness, willingness to report corruption and probability of corruption experience. It is possible that these variables may be associated with potential endogeneity or reverse causality problems. Hence, we decided to use the Internet connection in the household as instrumental variable in all regressions. This variable appears to be a suitable instrument, due to the fact that use of the Internet is highly correlated with Internet connection. This kind of positive correlation has been further tested in the paper. Internet connection alone should not have any direct effect on respondents' corruption awareness, information or they willingness to report corruption. The effect of Internet connection at level of individuals is mediated by Internet usage. Hence, the potential effect on corruption related variables can only be present via Internet usage. We also tested the strength of our instrument by using tests of weak instruments (for 2SLS regression) as described, for example, by Pflueger and Wang (2015). In all cases the tests show that the instrument is not weak and we are able to use it in the regressions. 
All regressions have estimated with robust standard errors. Due to the possibility that standard errors could be correlated at the country as well as within respondents' groups with the similar tolerance to corruption, we also used standard robust errors clustered by country and corruption tolerance group in several regressions.

In the first two groups of regressions we examine the potential determinants of respondents' knowledge about where to report corruption. We assume that using the Internet could have a positive effect on awareness about where to report corruption cases. Next, we analyse the potential effect variables capturing the influence of Internet use on the accuracy of respondents' corruption perception in each country. This could be also seen as respondents' awareness of corruption. In this case we compare the individuals' perception of corruption level in the country with observed average experience with corruption in the same country.

Finally, we examine potential relationships between Internet use and probability of experienced corruption as well as its reporting to public authorities or any other eligible body. Based on the theoretical background we assume that Internet usage could be negatively related to corruption experience and positively related to reporting of these cases.

The regressions also contain several socioeconomic independent control variables. The control variables have been included in line with the findings of previous studies as well as theoretical assumptions mentioned in the introduction. All variables are summarized and described in the Appendix.

The analysis is based on the data from the European commission (2014) Eurobarometer 79.1 survey. This survey was conducted in 2013 with altogether 27,752 respondents from all EU member states. In several cases we have to exclude missing observations and ambiguous answers from the sample, which actually reduced the total number of observations in some models. The average age of the respondents is 49.1 years. $46.3 \%$ of respondents are males and $53.7 \%$ of them are females. Approximately $69 \%$ of respondents have Internet access. While $50.4 \%$ of respondents use the Internet at home almost daily, $10.5 \%$ of them use the Internet at home two or three times a week. Furthermore, $23.3 \%$ of respondents use the Internet at work every day or almost every day. 
Turning to the problem of corruption and its reporting, the vast majority of respondents who experienced corruption did not report this fact to anyone. As it can be seen in Figure 1 those respondents who have some knowledge about where to report the corruption reported their experience with corruption more often than those who did not have this kind of knowledge. Hence, the very low level of corruption cases that have been reported could, perhaps, be to some extent the result of missing knowledge about where to report the corruption. Despite this fact, the share of those who report the corruption among those that have the knowledge remains small.

\section{<Insert Figure 1 here>}

Fig. 1 Share of respondents that reported and did not reported experienced corruption Source: Author's calculation based on the data from Eurobarometer 79.1.

Based on the data from Eurobarometer 79.1, approximately $49 \%$ of all respondents did not know where to report corruption. We further divide all respondents into two groups based on the Internet access ownership in the household. As we can see in Figure 2, those respondents who have Internet access at home seem to have more knowledge about where to report corruption when compared with those who do not have the Internet at home.

\section{<Insert Figure 2 here>}

Fig. 2 The distribution of answers to the question: "Do you know where to report the corruption?" Source: Author's calculation based on the data from Eurobarometer 79.1.

In accordance with the information function of the Internet, we also assume that Internet users have a more accurate view on the actual extent of corruption in their country. Moreover, as reported in the introductory section, Internet usage could have certain adverse effect on the level of the corruption itself. Suggested relationships between Internet usage and corruption related variables will be further examined in the next section. 


\section{Results and discussion}

In the first part of the analysis, we examine the potential effect of selected variables on respondents' knowledge about where to report corruption. The results of IV probit and probit regression models are shown in the Table 1. In addition to standard coefficients we also used marginal effect. We incorporated country-specific fixed effects in every second regression. The results suggest that there are several factors with statistically significant effects on the dependent variable and that Internet usage is among these. According to our results using the Internet at home seems to be already one of the key factors supporting the peoples' knowledge about corruption reporting. This variable is statically significant at $1 \%$ level in each regression model. Test of exogeneity yield slightly ambiguous results. We can say that the variable capturing Internet use at home seems to be endogenous only at $10 \%$ level of significance. Hence, we decided to use both IV probit as well as standard probit regression. Weak instrument tests prove the fact that the instrument is strong enough to be used in the regressions. We used several different models' specifications. However, results are similar for each of them. Based on the results we can also identify other significant factors, such as gender, age, education, level in the society and car ownership. Better educated people seem to have more information about where to report corruption. The same is true for men and those in the higher level of the society. The potential effect of age could be specified by inverse U-curve relationship. Retied people have, in general, less information about where to report corruption. The potential effect of the car ownership could be also interpreted as the consequence of respondents' higher affluence or mobility. 
Table 1

The results of IV probit and probit models - Know where to report corruption

\begin{tabular}{|c|c|c|c|c|c|c|}
\hline \multicolumn{7}{|c|}{$\begin{array}{c}\text { Dependent variable: Know where to report corruption }(\text { yes }=1, \text { no= }=0) \\
\text { Instrument: INTERNET ACCESS AT HOME }\end{array}$} \\
\hline \multirow[t]{2}{*}{ Estimation: } & \multicolumn{2}{|c|}{ IV Probit } & \multicolumn{4}{|c|}{ Probit } \\
\hline & $\begin{array}{c}\text { Coef. } \\
\text { (z-stat.) }\end{array}$ & $\begin{array}{c}\text { Coef. } \\
\text { (z-stat.) }\end{array}$ & $\begin{array}{c}\text { coef } \\
\text { (z-stat) }\end{array}$ & $\begin{array}{c}\mathrm{dy} / \mathrm{dx} \\
\text { (z-stat) }\end{array}$ & coef. & $\begin{array}{l}\text { dy/dx } \\
\text { (z-stat) }\end{array}$ \\
\hline $\begin{array}{l}\text { INTERNET USE AT } \\
\text { HOME (Instrumented in IV } \\
\text { Probit ) }\end{array}$ & $\begin{array}{c}0.04^{* * *} \\
(7.15)\end{array}$ & $\begin{array}{l}0.054^{* * *} \\
(8.95)\end{array}$ & $\begin{array}{l}0.033^{* * *} \\
(7.86)\end{array}$ & $\begin{array}{l}0.013^{* * *} \\
(7.86)\end{array}$ & $\begin{array}{l}0.046^{* * *} \\
(4.71)\end{array}$ & $\begin{array}{c}0.018^{* * *} \\
(4.71)\end{array}$ \\
\hline $\begin{array}{l}\text { GENDER } \\
(\mathrm{Male}=1, \text { Female }=0)\end{array}$ & $\begin{array}{c}0.204^{* * *} \\
(12.99)\end{array}$ & $\begin{array}{c}0.213^{* * *} \\
(13.42)\end{array}$ & $\begin{array}{c}0.205^{\star * *} \\
(13.03)\end{array}$ & $\begin{array}{c}0.082^{* * *} \\
(13.08)\end{array}$ & $\begin{array}{c}0.213^{* * *} \\
(10.52) \\
\end{array}$ & $\begin{array}{l}0.85^{\star * *} \\
(10.55)\end{array}$ \\
\hline AGE & $\begin{array}{c}0.023^{\star \star *} \\
(9.21)\end{array}$ & $\begin{array}{c}0.023^{\star \star \star} \\
(9.06)\end{array}$ & $\begin{array}{c}0.023^{\star \star \star} \\
(9.14) \\
\end{array}$ & $\begin{array}{c}0.009^{* \star *} \\
(9.14)\end{array}$ & $\begin{array}{c}0.023^{\star * *} \\
(4.41)\end{array}$ & $\begin{array}{c}0.009^{\star \star \star} \\
(4.41)\end{array}$ \\
\hline $\mathrm{AGE}^{2}$ & $\begin{array}{c}-0.0002^{* * *} \\
(-7.85)\end{array}$ & $\begin{array}{c}-0.0002^{* * *} \\
(-7.33)\end{array}$ & $\begin{array}{c}-0.0002^{* * *} \\
(-7.90)\end{array}$ & $\begin{array}{c}-0.0008^{* \star \star} \\
(-7.90)\end{array}$ & $\begin{array}{c}-0.0002^{* * *} \\
(-3.14)\end{array}$ & $\begin{array}{c}-0.0001^{* * *} \\
(-3.14)\end{array}$ \\
\hline EDUCATION & $\begin{array}{l}0.001^{*} \\
(1.82)\end{array}$ & $\begin{array}{c}0.002^{* *} \\
(2.13)\end{array}$ & $\begin{array}{l}0.001^{*} \\
(1.94)\end{array}$ & $\begin{array}{l}0.0005^{\star * *} \\
(1.94)\end{array}$ & $\begin{array}{l}0.001 \\
(1.58)\end{array}$ & $\begin{array}{c}0.0006^{* * *} \\
(1.58)\end{array}$ \\
\hline LIVING WITH PARTNER & $\begin{array}{l}-0.024 \\
(-1.38)\end{array}$ & $\begin{array}{l}-0.023 \\
(-1.29)\end{array}$ & $\begin{array}{l}-0.24 \\
(-1.37)\end{array}$ & $\begin{array}{c}-0.0097 \\
(-1.37)\end{array}$ & $\begin{array}{l}-0.022 \\
(-1.16)\end{array}$ & $\begin{array}{l}-0.008 \\
(-1.16)\end{array}$ \\
\hline SOCIETAL LEVEL & $\begin{array}{l}0.03^{\star \star \star} \\
(6.51)\end{array}$ & $\begin{array}{l}0.04^{\star \star \star} \\
(8.17)\end{array}$ & $\begin{array}{c}0.139^{\star \star \star} \\
(7.16)\end{array}$ & $\begin{array}{l}0.125^{\star \star *} \\
(6.95)\end{array}$ & $\begin{array}{c}0.042^{* * *} \\
(6.49) \\
\end{array}$ & $\begin{array}{c}0.017^{* * *} \\
(6.49) \\
\end{array}$ \\
\hline TV & $\begin{array}{l}-0.101^{*} \\
(-1.82)\end{array}$ & $\begin{array}{l}-0.085 \\
(-1.52) \\
\end{array}$ & $\begin{array}{l}-0.101^{*} \\
(-1.83)\end{array}$ & $\begin{array}{l}-0.040^{*} \\
(-1.84) \\
\end{array}$ & $\begin{array}{l}-0.082 \\
(-0.96) \\
\end{array}$ & $\begin{array}{l}-0.033 \\
(-0.96) \\
\end{array}$ \\
\hline CAR & $\begin{array}{c}0.132^{* \star *} \\
(6.70) \\
\end{array}$ & $\begin{array}{c}0.085^{\star \star *} \\
(4.10) \\
\end{array}$ & $\begin{array}{c}0.139^{\star \star *} \\
(7.16) \\
\end{array}$ & $\begin{array}{c}0.055^{\star \star *} \\
(7.17) \\
\end{array}$ & $\begin{array}{c}0.096^{* * *} \\
(3.84) \\
\end{array}$ & $\begin{array}{c}0.038^{* * *} \\
(3.84) \\
\end{array}$ \\
\hline RURAL/URBAN & $\begin{array}{l}0.005 \\
(0.53) \\
\end{array}$ & $\begin{array}{l}0.013 \\
(1.27)\end{array}$ & $\begin{array}{l}0.007 \\
(0.70)\end{array}$ & $\begin{array}{l}0.003 \\
(0.70)\end{array}$ & $\begin{array}{l}0.012 \\
(0.48)\end{array}$ & $\begin{array}{l}0.005 \\
(0.48)\end{array}$ \\
\hline UNEMPLOYED & $\begin{array}{c}0.04 \\
(1.40)\end{array}$ & $\begin{array}{l}0.034 \\
(1.18) \\
\end{array}$ & $\begin{array}{l}0.037 \\
(1.30) \\
\end{array}$ & $\begin{array}{l}0.015 \\
(1.31) \\
\end{array}$ & $\begin{array}{l}0.034 \\
(1.07) \\
\end{array}$ & $\begin{array}{l}0.012 \\
(1.07) \\
\end{array}$ \\
\hline RETIRED & $\begin{array}{c}-0.059^{\star *} \\
(-2.34) \\
\end{array}$ & $\begin{array}{l}-0.078^{\star * *} \\
(-3.02)\end{array}$ & $\begin{array}{l}-0.061^{* *} \\
(-2.40)\end{array}$ & $\begin{array}{c}-0.024^{* * *} \\
(-2.40)\end{array}$ & $\begin{array}{l}-0.09^{* * *} \\
(-2.22) \\
\end{array}$ & $\begin{array}{l}-0.036^{*} \\
(-2.22) \\
\end{array}$ \\
\hline Constant & $\begin{array}{l}-0.972^{* \star \star} \\
(-11.46)\end{array}$ & $\begin{array}{l}-9.24^{* * *} \\
(-8.86)\end{array}$ & $\begin{array}{l}-0.950^{\star \star \star} \\
(-11.33)\end{array}$ & & $\begin{array}{c}-1.177^{\star \star \star} \\
(-5.57)\end{array}$ & \\
\hline $\begin{array}{l}\text { Dummy variables for all EU } \\
\text { countries except Malta }\end{array}$ & Not included & Included & \multicolumn{2}{|c|}{ Not Included } & \multicolumn{2}{|c|}{ Included } \\
\hline Wald chi $^{2}$ & $638.5^{\star * *}$ & $1291.9^{* * *}$ & \multicolumn{2}{|c|}{$647.3^{\star * *}$} & & \\
\hline Log pseudolikelihood & -59520.6 & -58633.1 & \multicolumn{2}{|c|}{-17951.9} & \multicolumn{2}{|c|}{-17622.7} \\
\hline $\begin{array}{l}\text { Wald test of exogeneity } \\
\text { H0: variable is exogenous }\end{array}$ & $3.03^{*}$ & $3.14^{*}$ & & & & \\
\hline First-stage partial $\mathrm{R}^{2}$ & 0.589 & 0.561 & & & & \\
\hline First-stage F-statistics & $24924.4^{\star \star *}$ & $20374.8^{\star \star \star}$ & & & & \\
\hline $\begin{array}{l}\text { Weak instruments - critical } \\
\text { value (rejection rate }=10 \% \text { ) }\end{array}$ & 16.38 & 16.38 & & & & \\
\hline Standard errors: & \multicolumn{4}{|c|}{ Robuts S.E. } & \multicolumn{2}{|c|}{$\begin{array}{l}\text { Robuts S.E.clustered by } \\
\text { country }\end{array}$} \\
\hline Observations: & \multicolumn{6}{|c|}{26381} \\
\hline
\end{tabular}

Source: Author's calculations.

Note: (.) denotes Z-statistics and $* / * * / * * *$ denotes statistically significant at the 10/5/1 percent level.

In the next models we use slightly different variables capturing the usage of the Internet in order to further test the robustness of our results. This time we used binary independent variable not taking into the place of Internet usage. Hence, respondents could this time use the Internet at home, at work or anywhere else. The only thing that matters is the frequency of usage. We distinguish those who are 
using the Internet almost every day from those who are not. Despite the fact, that the instrumental variable seems to be slightly weaker in this case, it is still suitable according to the weak instrument tests. We again applied robust standard errors estimation as well as robust standard errors clustered by country.

As can be seen, the results in Table 2 again strongly suggest that those who using the Internet daily or almost daily are more aware of where to report corruption.

Table 2

The results of IV probit with different main independent variables - Know where to report corruption

Table 2

\begin{tabular}{|c|c|c|}
\hline \multicolumn{3}{|c|}{$\begin{array}{c}\text { Dependent variable: Know where to report corruption }(\mathrm{yes}=1, \mathrm{no}=0) \\
\text { Instrument: INTERNET ACCESS AT HOME }\end{array}$} \\
\hline Estimation: & IV Probit & IV Probit \\
\hline & $\begin{array}{c}\text { Coef. } \\
\text { (z-stat.) }\end{array}$ & $\begin{array}{c}\text { Coef. } \\
\text { (z-stat.) }\end{array}$ \\
\hline USE INTERNET DAILY- instrumented & $\begin{array}{l}1.917^{* \star *} \\
(9.30)\end{array}$ & $\begin{array}{l}1.917^{\star \star \star} \\
(2.83)\end{array}$ \\
\hline GENDER $($ Male= $=1$, Female $=0$ ) & $\begin{array}{l}0.207^{\star \star \star} \\
(13.14)\end{array}$ & $\begin{array}{l}0.207^{\star * *} \\
(11.95)\end{array}$ \\
\hline AGE & $\begin{array}{c}0.016^{\star \star \star} \\
(6.18)\end{array}$ & $\begin{array}{l}0.016^{\star \star} \\
(2.30)\end{array}$ \\
\hline $\mathrm{AGE}^{2}$ & $\begin{array}{c}-0.0002^{\star * \star} \\
(-6.67)\end{array}$ & $\begin{array}{c}-0.0002^{\star \star} \\
(-2.53)\end{array}$ \\
\hline EDUCATION & $\begin{array}{l}0.002^{* \star *} \\
(3.27)\end{array}$ & $\begin{array}{l}0.002^{* * *} \\
(2.64)\end{array}$ \\
\hline LIVING WITH PARTNER & $\begin{array}{l}-0.03^{*} \\
(-1.69)\end{array}$ & $\begin{array}{c}-0.03 \\
(-1.49)\end{array}$ \\
\hline SOCIETAL LEVEL & $\begin{array}{l}0.04^{* \star *} \\
(8.33)\end{array}$ & $\begin{array}{l}0.04^{* * *} \\
(5.66)\end{array}$ \\
\hline TV & $\begin{array}{l}-0.074 \\
(-1.36)\end{array}$ & $\begin{array}{l}-0.074 \\
(-0.91)\end{array}$ \\
\hline CAR & $\begin{array}{c}0.089^{\star \star *} \\
(4.37)\end{array}$ & $\begin{array}{c}0.089^{\star * \star} \\
(3.15)\end{array}$ \\
\hline RURAL/URBAN & $\begin{array}{l}0.015 \\
(1.50)\end{array}$ & $\begin{array}{l}0.015 \\
(0.61)\end{array}$ \\
\hline UNEMPLOYED & $\begin{array}{l}0.092^{* * *} \\
(3.09)\end{array}$ & $\begin{array}{l}0.092 \\
(1.35)\end{array}$ \\
\hline RETIRED & $\begin{array}{c}-0.075^{\star \star \star} \\
(-2.34)\end{array}$ & $\begin{array}{l}-0.075^{* *} \\
(-1.98)\end{array}$ \\
\hline Constant (coefficient and z-statistic): & $\begin{array}{l}-2.367^{\star \star \star} \\
(-11.96)\end{array}$ & $\begin{array}{l}-2.367^{* * *} \\
(-5.23)\end{array}$ \\
\hline Wald chi ${ }^{2}$ & $1517.8^{\star \star \star}$ & $2528660^{* * *}$ \\
\hline Log pseudolikelihood & -11272.2 & -11272.2 \\
\hline $\begin{array}{l}\text { Wald test of exogeneity } \\
\text { H0: variable is exogenous }\end{array}$ & $82.66^{\star * *}$ & $8.33^{\star * *}$ \\
\hline First-stage partial $\mathrm{R}^{2}$ & 0.03 & 0.03 \\
\hline First-stage F-statistics & $377.6^{\star \star \star}$ & $102.8^{\star \star \star}$ \\
\hline Standard errors & Robuts S.E. & $\begin{array}{c}\text { Robuts S.E.clustered } \\
\text { by country }\end{array}$ \\
\hline Observations: & 26381 & \\
\hline
\end{tabular}

Source: Author's calculations.

Note: (.) denotes z-statistics and $* / * * / * * *$ denotes statistically significant at the $10 / 5 / 1$ percent level. 
In the second part of our analysis, we focus our attention on the accuracy of the perceived extent of corruption in the country. The dependent variable is the absolute value of the difference between the level of corruption in the country as perceived by each respondent and the average experienced corruption in this country. The smaller the number the more accurate is the respondent's perception of the corruption in his or her country. Again, the role of information seems to be important in this case. This time we used 2SLS regression models with the same instruments as in previous cases. We also compare the results with those obtained by standard OLS regression. Frequency of Internet use at home as well as daily Internet usage have been applied as main independent variables. The results of regression models are summarized in Table 3. 
Table 3

The results of 2SLS and OLS regressions

\begin{tabular}{|c|c|c|c|c|c|}
\hline \multicolumn{6}{|c|}{$\begin{array}{l}\text { Dependent variable: The difference between perceived and actually observed corruption } \\
\text { Instrument: INTERNET ACCESS AT HOME }\end{array}$} \\
\hline \multirow{2}{*}{\multicolumn{2}{|c|}{ Estimation: }} & $\begin{array}{c}2 \text { SLS } \\
\text { (IV regression) }\end{array}$ & $\begin{array}{c}2 \mathrm{SLS} \\
\text { (IV regression) }\end{array}$ & OLS & OLS \\
\hline & & Coef. & Coef. & Coef. & Coef. \\
\hline \multicolumn{2}{|c|}{$\begin{array}{l}\text { INTERNET USE AT } \\
\text { HOME - instrumented }\end{array}$} & $\begin{array}{c}-0.006^{\star \star} \\
(-2.07)\end{array}$ & & $\begin{array}{l}-0.072^{* \star *} \\
(-3.40)\end{array}$ & $\begin{array}{l}-0.07^{\star \star \star} \\
(-2.29)\end{array}$ \\
\hline \multicolumn{2}{|c|}{$\begin{array}{l}\text { USE INTERNET DAILY- - } \\
\text { instrumented }\end{array}$} & & $\begin{array}{c}-0.227^{* *} \\
(-2.05)\end{array}$ & & \\
\hline \multicolumn{2}{|c|}{ GENDER $($ Male $=1$, Female=0) } & $\begin{array}{l}-0.043 \\
(-0.58)\end{array}$ & $\begin{array}{l}-0.005 \\
(-0.65)\end{array}$ & $\begin{array}{l}-0.04 \\
(-0.57)\end{array}$ & $\begin{array}{l}-0.04 \\
(-0.39)\end{array}$ \\
\hline \multicolumn{2}{|l|}{ AGE } & $\begin{array}{l}0.002 \\
(0.49)\end{array}$ & $\begin{array}{l}0.007^{\star *} \\
(2.29)\end{array}$ & $\begin{array}{l}0.0001 \\
(0.27)\end{array}$ & $\begin{array}{l}0.002 \\
(0.40)\end{array}$ \\
\hline \multicolumn{2}{|c|}{ EDUCATION } & $\begin{array}{c}0.00001 \\
(0.41)\end{array}$ & $\begin{array}{c}0.00003 \\
(0.07)\end{array}$ & $\begin{array}{l}0.0002 \\
(0.45)\end{array}$ & $\begin{array}{l}0.0002 \\
(0.40)\end{array}$ \\
\hline \multicolumn{2}{|c|}{ LIVING WITH PARTNER } & $\begin{array}{l}-0.013 \\
(-1.57)\end{array}$ & $\begin{array}{l}-0.012 \\
(-1.43)\end{array}$ & $\begin{array}{l}-0.013 \\
(-1.55)\end{array}$ & $\begin{array}{l}-0.013 \\
(-1.12)\end{array}$ \\
\hline \multicolumn{2}{|c|}{ SOCIETAL LEVEL } & $\begin{array}{l}0.002 \\
(0.93)\end{array}$ & $\begin{array}{l}0.002 \\
(0.76)\end{array}$ & $\begin{array}{l}0.002 \\
(1.03)\end{array}$ & $\begin{array}{l}0.002 \\
(0.52)\end{array}$ \\
\hline \multicolumn{2}{|l|}{$\mathrm{TV}$} & $\begin{array}{l}-0.021 \\
(-0.71)\end{array}$ & $\begin{array}{l}-0.022 \\
(-0.74)\end{array}$ & $\begin{array}{l}-0.021 \\
(-0.70)\end{array}$ & $\begin{array}{l}-0.021 \\
(-0.58)\end{array}$ \\
\hline \multicolumn{2}{|l|}{ CAR } & $\begin{array}{l}-0.009 \\
(-0.88)\end{array}$ & $\begin{array}{c}-0.01 \\
(-1.00)\end{array}$ & $\begin{array}{l}-0.007 \\
(-0.76)\end{array}$ & $\begin{array}{l}-0.007 \\
(-0.58)\end{array}$ \\
\hline \multicolumn{2}{|c|}{ RURAL/URBAN } & $\begin{array}{c}0.013^{* * *} \\
(2.73)\end{array}$ & $\begin{array}{c}0.013^{* * *} \\
(2.72)\end{array}$ & $\begin{array}{c}0.014^{* * \star} \\
(2.85)\end{array}$ & $\begin{array}{l}0.014 \\
(1.61)\end{array}$ \\
\hline \multicolumn{2}{|c|}{ UNEMPLOYED } & $\begin{array}{l}0.027^{* *} \\
(2.04)\end{array}$ & $\begin{array}{c}0.02 \\
(1.40)\end{array}$ & $\begin{array}{l}0.027^{* *} \\
(2.00)\end{array}$ & $\begin{array}{l}0.027 \\
(1.32)\end{array}$ \\
\hline \multicolumn{2}{|l|}{ RETIRED } & $\begin{array}{l}0.006 \\
(0.53)\end{array}$ & $\begin{array}{l}0.006 \\
(0.56)\end{array}$ & $\begin{array}{l}0.005 \\
(0.49)\end{array}$ & $\begin{array}{l}0.006 \\
(0.46)\end{array}$ \\
\hline \multicolumn{2}{|l|}{ Constant } & $\begin{array}{l}1.21^{\star \star \star} \\
(26.05)\end{array}$ & $\begin{array}{l}1.39^{\star \star \star} \\
(12.66)\end{array}$ & $\begin{array}{l}1.25^{\star \star \star} \\
(26.46)\end{array}$ & $\begin{array}{l}1.21^{\star \star \star} \\
(24.06)\end{array}$ \\
\hline \multicolumn{2}{|l|}{ Wald chi $^{2}$} & $14243.2^{* * *}$ & $14015.1^{* \star *}$ & $373.7^{\star \star \star}$ & \\
\hline \multicolumn{2}{|l|}{$\mathrm{R}^{2}$} & 0.297 & 0.294 & 0.297 & 0.297 \\
\hline \multirow{3}{*}{$\begin{array}{l}\text { H0: } \\
\text { variables } \\
\text { are } \\
\text { exogenous }\end{array}$} & Wu-Hausman test & $0.57(p$-value $=0.45)$ & 0.297 & & \\
\hline & Robust score chi ${ }^{2}$ & $0.61(p-$ value $=0.43)$ & $3.96^{* *}(p=0.047)$ & & \\
\hline & Result & $\begin{array}{c}\text { Exogenous at } 1 \% \\
\text { level }\end{array}$ & $\begin{array}{c}\begin{array}{c}\text { Endogenous at } 5 \% \\
\text { level }\end{array} \\
\end{array}$ & & \\
\hline \multicolumn{2}{|c|}{ First-stage partial $\mathrm{R}^{2}$} & 0.56 & 0.03 & & \\
\hline \multicolumn{2}{|c|}{ First-stage F-statistics: } & $20350.6^{* * *}$ & $364.5^{\star \star \star}$ & & \\
\hline \multicolumn{2}{|c|}{ Standard errors } & Robust S.E. & Robust S.E. & Robust S.E. & $\begin{array}{l}\text { Robust country } \\
\text { clustered S.E. }\end{array}$ \\
\hline \multicolumn{2}{|c|}{ Observations } & \multicolumn{4}{|c|}{26531} \\
\hline
\end{tabular}

Source: Author's calculations.

Note: (.) denotes z-statistics and $* / * * / * * *$ denotes statistically significant at the $10 / 5 / 1$ percent level. Standard errors have been corrected for heteroscedasticity.

As it can be seen, Internet use at home seems to be again statistically significant at least at $5 \%$ level. The same is true for the second variable, taking into account daily Internet use anywhere. The effect of both is negative, which implies that use of the Internet helps respondents to better evaluate the 
incidence of the corruption in the country. Hence, the frequency of Internet usage seems to be an important factor affecting the accuracy of corruption perception. Interestingly, similar relationship could not be seen in the case of TV or car ownership.

Based on these findings, we can say that the use of the Internet plays a significant role in knowledge about corruption and its reporting. Hence, it would seem to be a useful tool to increase the corruption awareness. In some ways, similar results have been obtained by Goel, Nelson and Naretta (2012). The authors stated that the Internet has a negative effect on corruption by increasing the corruption awareness via its information function. In line with this, it seems likely that the Internet could be also beneficial in reducing the corruption itself. We can assume that those using the Internet often are less often experiencing corruption due to several facts. Firstly, they should be able to use electronic communication with public authorities more often, which could in theory reduce exposure to corruption. Secondly, due to better awareness about corruption and potential sanctions they could more frequently avoid corruption. In line with previous results we assume that those using the Internet more often are also more prone to report corruption attempt. We again tested these assumptions using IV probit regressions with instrumental variables. In this case we also included tolerance to corruption as well as contact with public authorities as independent variables. Moreover, standard errors have been clustered based on the corruption tolerance group in the two regression models, which allowed us to control for heterogeneity of corruption perception in different groups of respondents. We also use standard errors clustered at the country level as in some of the previous regressions. However, in this case the results do not support our assumption about the potential negative effect of Internet usage on respondents' experience of corruption. Our main independent variable is not statistically significant. On the other hand, as expected we found evidence for the positive correlation between corruption tolerance and corruption experience. Those having higher tolerance to corruption tend to have more experience with corruption. This is complementary to results of previous studies (e.g. Hunady, 2017). Similarly, having more frequent contact with public authorities is significantly and positively correlated with corruption experience. 
Table 4

Results of IV probit regressions using experienced corruption and reporting corruption as dependent variables

\begin{tabular}{|c|c|c|c|c|c|c|}
\hline & \multicolumn{3}{|c|}{$\begin{array}{c}\text { Dependent variable: } \\
\text { EXPERIENCED CORRUPTION }\end{array}$} & $\begin{array}{r}\text { Deper } \\
\text { REPORT } \\
\text { (sample restric } \\
\text { experienced o }\end{array}$ & $\begin{array}{l}\text { dent varia } \\
\text { D CORRL } \\
\text { ted limited } \\
\text { r witnessec }\end{array}$ & $\begin{array}{l}\text { ble: } \\
\text { JPTION } \\
\text { to those who } \\
\text { d corruption) }\end{array}$ \\
\hline \multirow[t]{2}{*}{ Estimation: } & \multicolumn{3}{|c|}{ IV probit } & \multicolumn{3}{|c|}{ IV probit } \\
\hline & \multicolumn{3}{|c|}{ Coef. } & \multicolumn{3}{|c|}{ Coef. } \\
\hline $\begin{array}{l}\text { INTERNET USE AT } \\
\text { HOME - instrumented }\end{array}$ & $\begin{array}{l}0.014 \\
(0.59)\end{array}$ & $\begin{array}{l}0.015 \\
(1.48)\end{array}$ & $\begin{array}{l}-0.018 \\
(-1.39)\end{array}$ & $\begin{array}{l}0.11^{\star *} \\
(2.42)\end{array}$ & $\begin{array}{l}0.11^{* * *} \\
(3.73)\end{array}$ & $\begin{array}{l}0.103^{* * *} \\
(3.28)\end{array}$ \\
\hline $\begin{array}{l}\text { CORRUTPION } \\
\text { TOLERANCE }\end{array}$ & & $\begin{array}{l}0.247^{\star \star *} \\
(10.72)\end{array}$ & $\begin{array}{c}0.386^{* * *} \\
(6.65)\end{array}$ & & & \\
\hline \begin{tabular}{|l|} 
CONTACT WITH \\
PUBLIC AUTHORITIES
\end{tabular} & $\begin{array}{c}0.871^{\star * *} \\
(5.67)\end{array}$ & $\begin{array}{l}0.867^{\star \star *} \\
(17.12)\end{array}$ & $\begin{array}{c}0.771^{* * *} \\
(6.70)\end{array}$ & & & \\
\hline $\begin{array}{l}\text { GENDER }(\text { Male }=1, \\
\text { Female }=0)\end{array}$ & $\begin{array}{c}0.114^{* * *} \\
(7.87)\end{array}$ & $\begin{array}{c}0.114^{* * \star} \\
(4.39)\end{array}$ & $\begin{array}{c}0.96 \\
(3.65)\end{array}$ & $\begin{array}{l}-0.004 \\
(-0.10)\end{array}$ & $\begin{array}{l}-0.005 \\
(-0.06)\end{array}$ & $\begin{array}{l}0.002 \\
(0.02)\end{array}$ \\
\hline AGE & $\begin{array}{l}0.021^{\star \star \star} \\
(2.97)\end{array}$ & $\begin{array}{c}0.023^{\star \star \star} \\
(4.50)\end{array}$ & $\begin{array}{l}0.28^{* \star \star} \\
(5.98)\end{array}$ & $\begin{array}{l}0.003 \\
(1.04)\end{array}$ & $\begin{array}{l}0.003 \\
(0.86)\end{array}$ & $\begin{array}{c}0.025^{\star \star *} \\
(2.03)\end{array}$ \\
\hline $\mathrm{AGE}^{2}$ & $\begin{array}{c}-0.0003^{* * *} \\
(-3.72)\end{array}$ & $\begin{array}{l}-0.0003^{\star * *} \\
(-5.08)\end{array}$ & $\begin{array}{l}-0.0004 \\
(-6.89)\end{array}$ & & & $\begin{array}{c}-0.0002^{*} \\
(-1.81)\end{array}$ \\
\hline EDUCATION & $\begin{array}{c}0.003^{* * *} \\
(4.07)\end{array}$ & $\begin{array}{l}0.003^{* *} \\
(2.57)\end{array}$ & $\begin{array}{l}0.003 \\
(1.84)\end{array}$ & $\begin{array}{l}0.003 \\
(1.04)\end{array}$ & $\begin{array}{l}0.003 \\
(0.97)\end{array}$ & $\begin{array}{l}0.003 \\
(0.92)\end{array}$ \\
\hline $\begin{array}{l}\text { LIVING WITH A } \\
\text { PARTNER }\end{array}$ & $\begin{array}{l}0.039 \\
(0.94)\end{array}$ & $\begin{array}{l}0.036 \\
(1.21)\end{array}$ & $\begin{array}{l}0.065 \\
(1.15)\end{array}$ & $\begin{array}{l}0.081^{\star *} \\
(1.98)\end{array}$ & $\begin{array}{l}0.081 \\
(1.01)\end{array}$ & $\begin{array}{l}0.052 \\
(0.65)\end{array}$ \\
\hline SOCIETAL LEVEL & $\begin{array}{l}-0.011 \\
(-1.40) \\
\end{array}$ & $\begin{array}{l}-0.009 \\
(-1.04) \\
\end{array}$ & $\begin{array}{l}-0.019 \\
(-1.78)\end{array}$ & $\begin{array}{l}0.016 \\
(1.06)\end{array}$ & $\begin{array}{l}0.017 \\
(0.75)\end{array}$ & $\begin{array}{c}0.02 \\
(1.23)\end{array}$ \\
\hline TV & $\begin{array}{l}-0.021 \\
(-0.26)\end{array}$ & $\begin{array}{l}-0.036 \\
(-0.38)\end{array}$ & $\begin{array}{l}0.075 \\
(0.66)\end{array}$ & $\begin{array}{c}0.06 \\
(0.26)\end{array}$ & $\begin{array}{l}0.064 \\
(0.28)\end{array}$ & $\begin{array}{l}0.045 \\
(0.20)\end{array}$ \\
\hline CAR & $\begin{array}{l}0.016 \\
(0.19)\end{array}$ & $\begin{array}{l}0.012 \\
(0.34)\end{array}$ & $\begin{array}{l}-0.105 \\
(-2.04)\end{array}$ & $\begin{array}{l}-0.018 \\
(-0.75)\end{array}$ & $\begin{array}{l}-0.018 \\
(-0.19)\end{array}$ & $\begin{array}{l}-0.035 \\
(-0.34)\end{array}$ \\
\hline RURAL/URBAN & $\begin{array}{l}0.016 \\
(1.32)\end{array}$ & $\begin{array}{l}0.014 \\
(0.86)\end{array}$ & $\begin{array}{l}0.031 \\
(0.70)\end{array}$ & $\begin{array}{l}-0.051 \\
(-0.78)\end{array}$ & $\begin{array}{l}-0.051 \\
(-1.09)\end{array}$ & $\begin{array}{l}-0.049 \\
(-0.69)\end{array}$ \\
\hline UNEMPLOYED & $\begin{array}{c}0.137^{\star \star} \\
(1.98)\end{array}$ & $\begin{array}{l}0.081^{*} \\
(1.81)\end{array}$ & $\begin{array}{l}0.045 \\
(0.70)\end{array}$ & $\begin{array}{c}0.359^{* \star *} \\
(4.87)\end{array}$ & $\begin{array}{c}0.359^{\star * \star} \\
(3.16)\end{array}$ & $\begin{array}{l}0.35^{\star \star *} \\
(2.83)\end{array}$ \\
\hline RETIRED & $\begin{array}{c}0.137^{\star \star \star} \\
(3.39)\end{array}$ & $\begin{array}{l}0.14^{* \star *} \\
(2.95)\end{array}$ & $\begin{array}{l}0.148 \\
(1.60)\end{array}$ & $\begin{array}{c}0.196^{* * *} \\
(4.57)\end{array}$ & $\begin{array}{c}0.196^{\star \star *} \\
(1.54)\end{array}$ & $\begin{array}{l}0.253^{*} \\
(1.86)\end{array}$ \\
\hline Constant & $\begin{array}{l}-3.18^{\star * *} \\
(-51.48)\end{array}$ & $\begin{array}{l}-3.54^{* \star \star} \\
(-17.04)\end{array}$ & $\begin{array}{l}-3.19^{* * *} \\
(-15.03)\end{array}$ & & $\begin{array}{l}-2.1^{* * *} \\
(-5.05)\end{array}$ & $\begin{array}{l}-2.49 \\
(-6.61)\end{array}$ \\
\hline $\begin{array}{l}\text { Dummy variables for } \\
27 \text { countries }\end{array}$ & Included & Included & Not included & Included & Included & Included \\
\hline Wald chi ${ }^{2}$ & $9.82^{* * *}$ & $1230.0^{* * *}$ & $205.48^{\star \star *}$ & $5.14^{*}$ & $211.6^{* * *}$ & $6.05 e+08^{* * *}$ \\
\hline Log pseudolikelihood & -48924.4 & -48868.1 & -49879 & -5326.3 & -5326.3 & -5322.6 \\
\hline $\begin{array}{l}\text { Wald test of exogeneity } \\
\text { H0: variable is exogenous }\end{array}$ & $\begin{array}{c}2.64 \\
(p=0.104)\end{array}$ & $\begin{array}{c}5.85^{\star *} \\
(p=0.015)\end{array}$ & $\begin{array}{c}3.40^{*} \\
(p=0.065)\end{array}$ & $\begin{array}{c}1.39 \\
(p=0.238)\end{array}$ & $\begin{array}{c}5.77^{* *} \\
p=0.02\end{array}$ & $\begin{array}{c}5.79^{\star \star} \\
p=0.016\end{array}$ \\
\hline First-stage partial $\mathrm{R}^{2}$ & 0.56 & 0.56 & 0.59 & 0.59 & 0.59 & 0.59 \\
\hline First-stage F-statistics: & $1865.0^{* * *}$ & $2137.6^{\star \star \star}$ & $25900.5^{\star * \star}$ & $2307.4^{* * *}$ & $2642.3^{\star \star *}$ & $780.1^{* * *}$ \\
\hline Standard errors: & $\begin{array}{c}\text { Robuts } \\
\text { S.E.clustered by } r \\
\text { corruption } \\
\text { tolerance group }\end{array}$ & Robuts S.E. & $\begin{array}{l}\text { Robuts } \\
\text { S.E.clustered by } \\
\text { country }\end{array}$ & \begin{tabular}{|c|} 
Robuts \\
S.E.clustered by \\
corruption \\
tolerance group
\end{tabular} & $\begin{array}{l}\text { Robust } \\
\text { S.E. }\end{array}$ & $\begin{array}{l}\text { Robuts } \\
\text { S.E.clustered } \\
\text { by country }\end{array}$ \\
\hline Observations & & 27752 & & & 3022 & \\
\hline
\end{tabular}

Source: Author's calculations.

Note: (.) denotes z-statistics and $* / * * / * * *$ denotes statistically significant at the $10 / 5 / 1$ percent level. Standard errors have been corrected for heteroscedasticity. 
Furthermore, taking into account only those respondents that have some experience with corruption we found that those using the Internet more often are significantly more prone to report experienced cases of corruption. This effect has a notably high level of statistical significance. Thus, despite the fact that Internet usage seems to have no measurable effect on experiencing corruption, it does have significant an effect on the behaviour of participants. Internet users are more likely to report corruption cases and this could be also related to having better corruption awareness and knowledge about where to report such a case.

Our results suggest that use of the Internet by citizens has certain benefits with respect to reducing the corruption in a country. Given this, what might be done to increase Internet usage? The basic requirement is an Internet connection. We therefore decided to look briefly at the main reasons why there are still many people without an Internet connection in many countries. The most important reasons can be identified from the responses to another question in Eurobarometer 79.1 survey. The question has been stated as follows: "From the following list, which best explain why your household does not have access to the Internet?"

The results are presented graphically in Figure 3. As can be seen, cost is reported as by far the most significant obstacle to the acquisition of Internet access followed by the lack of interest among respondents and their family members. Lack of knowledge and concerns about the access to unsuitable content are two other factors that are worth noting. On the other hand, the coverage of Internet network infrastructure seems to be in general sufficient enough in the EU, because only a few respondents see this as the obstacle to acquiring or maintaining an Internet connection.

\section{$<$ Insert Figure 3 here>}

Fig. 3 The most important reasons why the households do not have Internet access. Source: Author's calculation based on the data from Eurobarometer 79.1.

When we look in more detail at costs, there are four different types of cost mentioned in the questionnaire. The results are shown in Figure 4. 
<Insert Figure 4 here>

Fig. 4 Individual cost aspects and their importance

Author's calculation based on the data from Eurobarometer 79.1.

As can be seen, the initial installation cost is often the most challenging problem for households. In contrast, both types of subscription cost as well as the cost of buying the necessary hardware are considered by respondents to be less significant.

Finally, it is important to notice that the approach used in this study has some limitations and potential drawbacks. The approach used allows us to analyse corruption based on a considerable number of observations at the level where this problem actually arises. Despite this fact, it is important to notice that survey data have some limitation when studying corruption and its determinants. Firstly, perception based data can be slightly (Olken 2009; Heywood and Rose 2014). In line with this fact, we are also using some experience-based measures in our analysis. Secondly, it has to be borne in mind that this analysis is only a static one; consequently thus there are no trends captured here.

\section{Conclusions and policy implication}

Our results in general confirm the importance of the Internet and its usage with respect to corruption related variables. We used IV probit and 2SLS regressions in order to eliminate the problem of potential endogeneity. According to the data from Eurobarometer, less than $10 \%$ of respondents who experienced corruption reported this experience to someone else. Furthermore, nearly half of respondents in the sample actually don't know where to report it. Our analysis provides new empirical evidence about the positive effect of Internet usage on the respondents' knowledge about where to report a case of corruption. This effect is positive for using the Internet and its frequency. On the other hand, this kind of effect could not be detected, for example, in the case of TV. It is likely that the Internet is at least in this case a much useful source of the type of information necessary to reduce corruption. Those who use the Internet daily have, in general, significantly more knowledge about corruption reporting. Moreover, Internet usage appears to have significant effect also on actual 
reporting of experiences of corruption. This could be of course related to having better knowledge about corruption reporting. Gender, age, education and societal level are also other significant factors affecting the knowledge about where to report corruption. Despite this, these indicators seem not to be significantly related to the actual willingness of respondents to report experienced corruption. On the other hand, we fail to find any significant effect of Internet usage on experience of corruption. We infer that those using the Internet frequently are not a group which experiences a lower incidence of corruption. However, they are in general more open to reporting experienced corruption cases. This can also mean that they are rather victims then offenders.

In line with the information function of the Internet we decide to test the effect of Internet usage on accuracy of respondents' corruption perception in the country. As far as we know, this kind of causality has not been examined in the literature so far. We calculated the difference between the subjectively perceived extent of corruption in the country and the average reported experience with corruption in this country. Our results strongly suggest that use of the Internet is one of the factors significantly enhancing the corruption awareness.

Based on our results, we can say that Internet usage improve corruption awareness as well the knowledge about where to report the corruption and also actual frequency of corruption reporting. All these facts could play important role in the effort to reduce the corruption. Internet access is of course the primary precondition of Internet usage. Expanding Internet access can certainly deliver many benefits; some of them are related to anti-corruption policies. Decision/policy making bodies should be aware of this fact.

An increase in Internet access is, therefore, a possible anti-corruption tool. Thus, what could be done in particular to increase the Internet access? The cost aspect in general proves to be the most pressing problem for the households. To be more concrete, especially the initial installation cost seems to be the most significant obstacle. The results with regard to cost suggest that a direct one-off subsidy for setting up an Internet connection could be the most effective way to promote Internet access, that the cost of the necessary hardware is probably not a decisive factor for most of the people in their decision whether or not to use the Internet and that Internet network coverage appears to be adequate in the most areas covered by the survey. 


\section{Acknowledgement}

We would like to thank anonymous reviewers for their valuable comments and suggestions for the manuscript.

\section{References}

[1] Andrés, A. R., \& Goel, R. K. (2011). Corruption and software piracy: A comparative perspective. Policy \& Internet, 3(3), 1-22. DOI: 10.2202/1944-2866.1088.

[2] Andersen, T. B. (2009). E-Government as an anti-corruption strategy. Information Economics and Policy, 21(3), 201-210. DOI: 10.1016/j.infoecopol.2008.11.003.

[3] Andersen, T. B., Bentzen, J., Dalgaard, C. J. \& Selaya, P. (2011). Does the Internet Reduce Corruption? Evidence from US States and across countries. The World Bank Economic Review, 25(3), 387-417.

[4] Ata, A. Y. \& Arvas, M. A. (2011). Determinants of economic corruption: a cross-country data analysis. International Journal of Business and Social Science, 2(13), 161-169.

[5] Bhattacharyya, S. \& Hodler, R. (2015). Media freedom and democracy in the fight against corruption. European Journal of Political Economy, 39, 13-24.

DOI:10.1016/j.ejpoleco.2015.03.004

[6] Brunetti, A. \& Weder, B. (2003). A free press is bad news for corruption. Journal of Public economics, 87(7), 1801-1824. .

[7] Choi, J. W. (2014). E-government and corruption: A cross-country survey. World Political Science, 10(2), 217-236. DOI: 10.1515/wpsr-2014-0012.

[8] Dirienzo, C. E., Das, J., Cort, K.\& Burbridge, J. (2007). Corruption and the Role of Information. Journal of International Business Studies, 38(2), 320 - 332.

[9] Dreher, A., Kotsogiannis, C. \& McCorriston, S. (2009). How do institutions affect corruption and the shadow economy? International Tax and Public Finance, 16(6), 773-796.

[10] Garcia-Murillo, M. (2010). The effect of Internet access on government corruption. Electronic Government, An International Journal, 7(1), 22-40. 
[11] Goel, R. K., Nelson, M. A. \& Naretta, M. A. (2012). The Internet as an indicator of corruption awareness. European Journal of Political Economy, 28(1), pp. 64-75.

[12] Goel, R. K. \& Ram, R. (2013)., Economic uncertainty and corruption: Evidence from a large cross-country data set. Applied economics, 45(24), 3462-3468. DOI:

$10.1080 / 00036846.2012 .714073$

[13] Elbahnasawy, N. G. (2014). E-government, internet adoption, and corruption: An empirical investigation. World Development, 57, 114-126.

[14] Elbahnasawy, N. G. \& Revier, C. F. (2012). The Determinants of Corruption: CrossCountry- Panel- Data Analysis. The Developing Economies, 50(4), 311-333. DOI: $10.1111 / j .1746-1049.2012 .00177 . x$

[15] European Commission. (2013). Eurobarometer 79.1: E-Communications in the Household and Corruption, February-March 2013. Cologne, Germany: GESIS/Ann Arbor, MI: Interuniversity Consortium for Political and Social Research. DOI: http://doi.org/10.3886/ICPSR35083.v1

[16] Hunady, J. \& Orviska, M. (2015). Does the Internet usage reduce the corruption in public sector? - The short run and long run causality. Acta Aerarii Publici, 12(1), 22-43.

[17] Hunady, J. (2017). Individual and institutional determinants of corruption in the EU countries: the problem of its tolerance. Economia Politica, 34(1), 139-157. DOI: https://doi.org/10.1007/s40888-017-0056-4.

[18] Im, T., Cho, W., Porumbescu, G.\& Park, J.( 2014). Internet, trust in government, and citizen compliance. Journal of Public Administration Research and Theory, 24(3), 741-763. DOI: 10.1093/jopart/mus037.

[19] Ionescu, L. (2015). The Role of E-Government in Curbing the Corruption in Public Administration. Economics, Management, and Financial Markets, (1), 48-53.

[20] Iwasaki, I. \& Suzuki, T. (2012). The determinants of corruption in transition economies. Economics Letters, 114(1), 54-60. DOI:10.1016/j.econlet.2011.08.016. 
[21] Kalenborn, C. \& Lessmann, C. (2013). The impact of democracy and press freedom on corruption: Conditionality matters. Journal of Policy Modeling, 35(6), 857-886. DOI:10.1016/j.jpolmod.2013.02.009.

[22] Kim, C. K. (2014). Anti-corruption initiatives and e-government: a cross-national study. Public Organization Review, 14(3), 385-396. DOI: 10.1007/s11115-013-0223-1.

[23] Lehdonvirta, V. \& Bright, J. (2015). Crowdsourcing for public policy and government. Policy \& Internet, 7(3), 263-267. DOI: 10.1002/poi3.103.

[24] Lio, M. C., Liu, M. C. \& Ou, Y. P. (2011). Can the Internet reduce corruption? A crosscountry study based on dynamic panel data models. Government Information Quarterly, 28(1), 47-53. DOI:10.1016/j.giq.2010.01.005.

[25] Mocan, N. (2008). What determines corruption? International evidence from microdata. Economic Inquiry, 46(4), 493-510. DOI: 10.1111/j.1465-7295.2007.00107.x.

[26] Newey, W. K. (1987). Efficient estimation of limited dependent variable models with endogenous explanatory variables. Journal of Econometrics, vol. 36, 231-250.

[27] Orviska, M. \& Hudson, J. (2009). Dividing or uniting Europe? Internet usage in the EU. Information Economics and Policy, vol. 21, issue 4, 279-290. DOI:10.1016/j.infoecopol.2009.06.002.

[28] Pflueger, C. E. \& Wang S. (2015). A robust test for weak instruments in Stata. The Stata Journal, vol. 15, issue 1, 216-225.

[29] Rehman, H. U. \& Naveed, A. (2007). Determinants of Corruption and its Relation to GDP A panel study. Journal of Political Studies, 7, 27-59.

[30] Rivas, M. F. (2013). An experiment on corruption and gender. Bulletin of Economic Research, 65(1), 10-42.

[31] Shim, D. C., \& Eom, T. H. (2008). E-government and anti-corruption: Empirical analysis of international data. International Journal of Public Administration, 31(3), 298-316.

[32] Serra, D. (2006). Empirical determinants of corruption: A sensitivity analysis. Public Choice, 126, 225-256. DOI: 10.1007/s11127-006-0286-4. 
[33] Sööt, M.L. \& Rootalu, K. (2012). Institutional trust and opinions of corruption. Public Administration and Development, 32(1), 82-95. DOI: 10.1002/pad.616.

[34] Torgler, B. \& Valev, N. T. (2010). Gender and public attitudes toward corruption and tax evasion. Contemporary Economic Policy, 28(4), 554-568. DOI: 10.1111/j.14657287.2009.00188.x.

[35] Zhao, X., \& Xu, H.D. (2015). E-Government and Corruption: A Longitudinal Analysis of Countries. International Journal of Public Administration, 38(6), 1-12. doi:

$10.1080 / 01900692.2014 .942736$.

[36] Zinnbauer, D. (2015). Crowdsourced Corruption Reporting: What Petrified Forests, Street Music, Bath Towels, and the Taxman Can Tell Us About the Prospects for Its Future. Policy \& Internet, 7(1), 1-24. DOI: 10.1002/poi3.84.

\section{Appendix}

Description of socioeconomic variables included in the regressions

\begin{tabular}{|c|c|}
\hline \multicolumn{2}{|c|}{ All data are retrieved from Eurobarometer 79.1 (2013): E-Communications in the Household and Corruption } \\
\hline Variable - label & Description of dependent variables and their coding \\
\hline $\begin{array}{l}\text { KNOW WHERE TO } \\
\text { REPORT } \\
\text { CORRUPTION }\end{array}$ & $\begin{array}{l}\text { If you were to experience or witness a case of corruption, would you know where to } \\
\text { report it to?: Yes }=1 ; \text { No }=2 \text {. (don't know answers excluded) }\end{array}$ \\
\hline $\begin{array}{l}\text { THE DIFFERENCE } \\
\text { BETWEEN } \\
\text { PERCEIVED AND } \\
\text { OBSERVED } \\
\text { CORRUPTION }\end{array}$ & 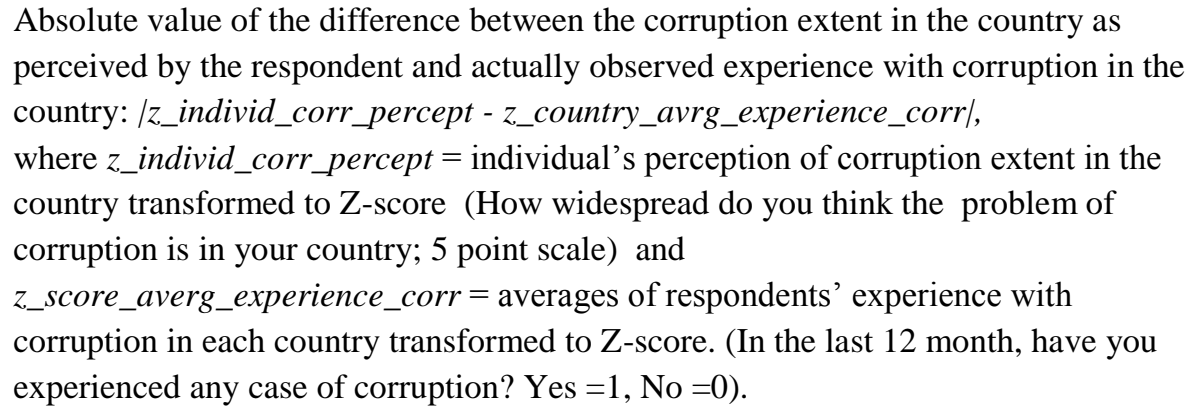 \\
\hline $\begin{array}{l}\text { EXPERIENCED } \\
\text { CORRUPTION }\end{array}$ & $\begin{array}{l}\text { In the last } 12 \text { months, have you experienced any case of corruption? } \\
\text { (Yes, experienced }=1 ; \text { No }=0 \text { ) }\end{array}$ \\
\hline $\begin{array}{l}\text { INTERNET ACCESS } \\
\text { AT HOME }\end{array}$ & $\begin{array}{l}\text { Which of the following goods do you have?...An Internet connection at home } \\
(\text { yes }=1 ; \text { no }=0 \text { ) - used as the instrument }\end{array}$ \\
\hline $\begin{array}{l}\text { INTERNET USE AT } \\
\text { HOME }\end{array}$ & $\begin{array}{l}\text { Could you tell me if you use the Internet at home (Everyday/almost every day }=6, \\
\text { two or three times a week }=5 \text {, about once a week }=4 \text {, two or three times a month }=3 \text {, } \\
\text { less often }=2, \text { never or no Internet access }=1 \text { ) }\end{array}$ \\
\hline $\begin{array}{l}\text { INTERNET USE } \\
\text { EVERY DAY - }\end{array}$ & $\begin{array}{l}\text { Respondent is using internet at home, at work or anywhere else everyday/almost } \\
\text { every day coded as } 1 \text { else coded as } 0\end{array}$ \\
\hline $\begin{array}{l}\text { INTERNET USE AT } \\
\text { LEAST ONE TIME A } \\
\text { WEEK }\end{array}$ & $\begin{array}{l}\text { Respondent is using internet at home, at work or anywhere else at least one time a } \\
\text { week coded as } 1 \text { else coded as } 0\end{array}$ \\
\hline
\end{tabular}




\begin{tabular}{|l|l|}
\hline $\begin{array}{l}\text { CORRUPTION } \\
\text { TOLERANCE }\end{array}$ & $\begin{array}{l}\text { If you wanted to get something from the public administration or public services, to } \\
\text { what extent do you think it is acceptable to do any of the following? To give money, } \\
\text { 2. To give a gift, 3. To do a favour. Tolerance is calculated. Based on responses } \\
\text { (recoded to binary variable: Acceptable or Tolerated =1; Unacceptable= })\end{array}$ \\
\hline $\begin{array}{l}\text { CONTACT WITH } \\
\text { PUBLIC } \\
\text { AUTHORITIES }\end{array}$ & $\begin{array}{l}\text { Over the last } 12 \text { months, have you had any contact with any of the following: public } \\
\text { authorities... (yes=1, no=0) }\end{array}$ \\
\hline MALE & Respondent's gender: Male $=1 ;$ Female $=0$ \\
\hline AGE & How old are you? (exact age) \\
\hline EDUCATION & How old were you when you stopped full-time education? (exact age) \\
\hline $\begin{array}{l}\text { LIVING WITH } \\
\text { PARTNER }\end{array}$ & Married or living in the partnership: Yes=1, No=0 \\
\hline SOCIETAL LEVEL & Self 10 point scale; lowest level=1, highest level $=10$ ) \\
\hline TV & Which of the following goods do you have?...Television (yes=1; no=0) \\
\hline CAR & Which of the following goods do you have?...Car (yes=1; no=0) \\
\hline RURAL/URBAN & $\begin{array}{l}\text { Would you say you live in a...? Rural area or village }=1 ; \text { Small or middle sized town } \\
=2 ; \text { Large town }=3\end{array}$ \\
\hline UNEMPLOYED & What is your current occupation?..Unemployed or temporarily not working (yes=1) \\
\hline RETIRED & What is your current occupation?...Retired or unable to work through illness (yes=1) \\
\hline
\end{tabular}

Figure 1

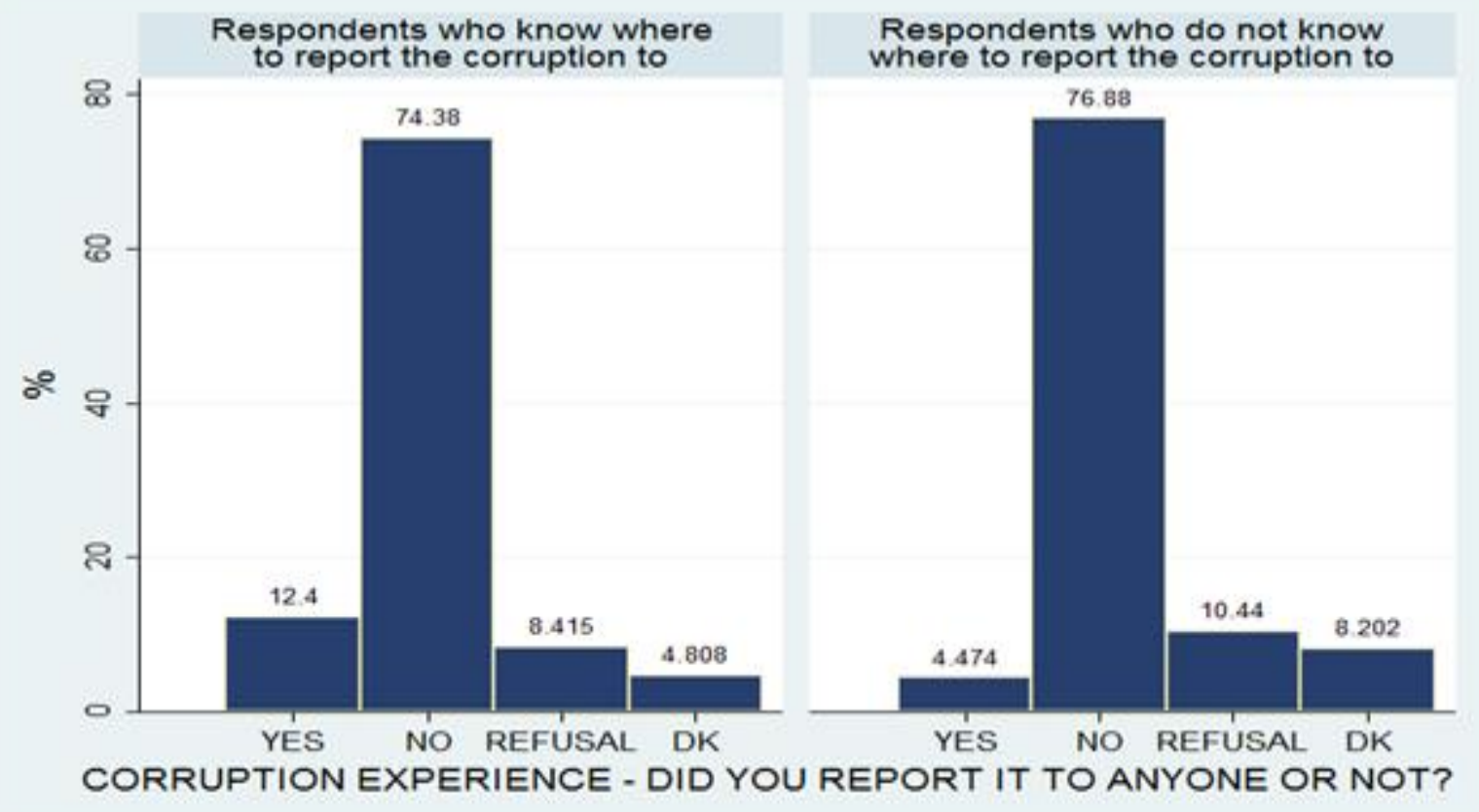


Figure 2

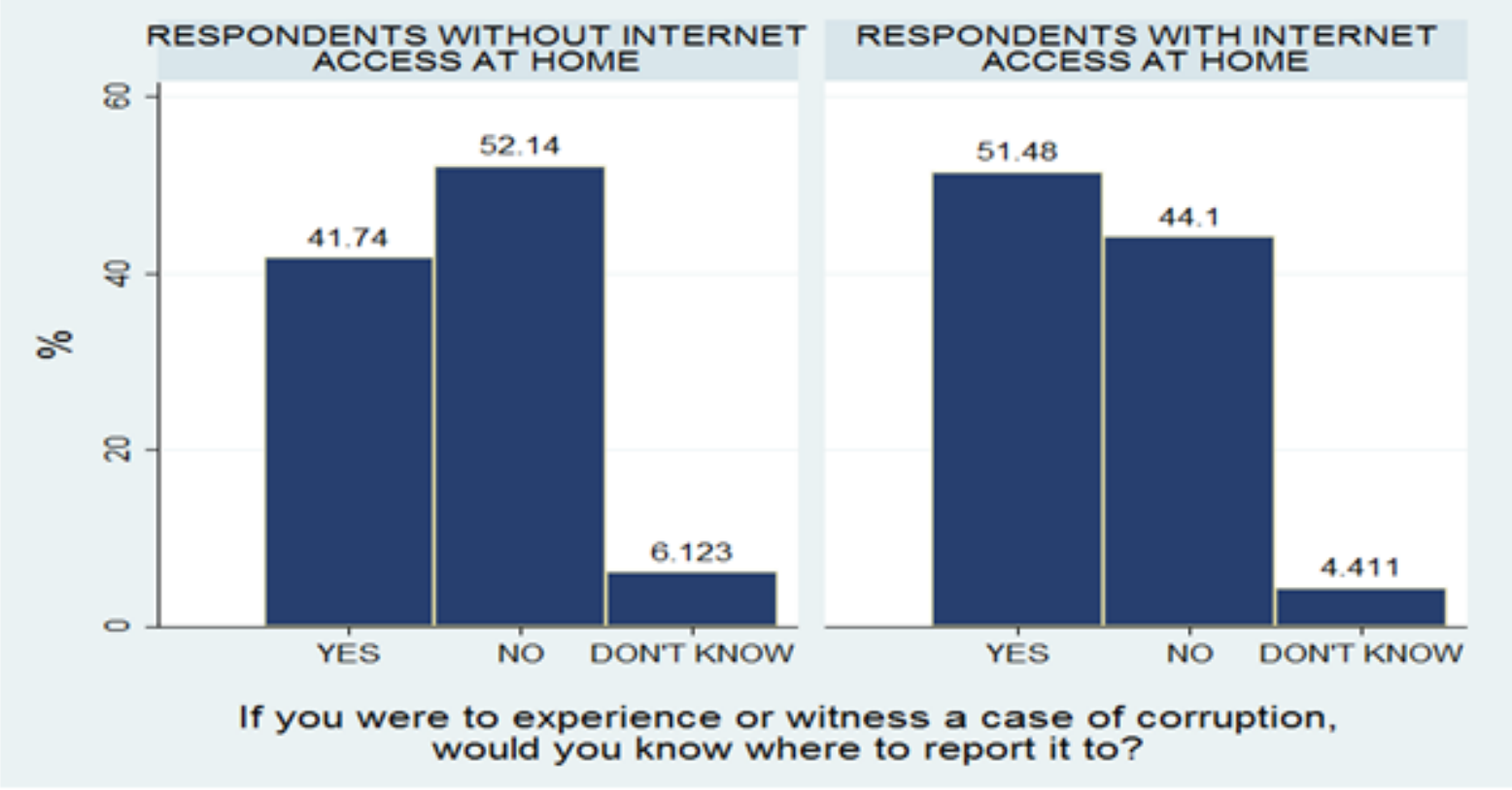

Figure 3

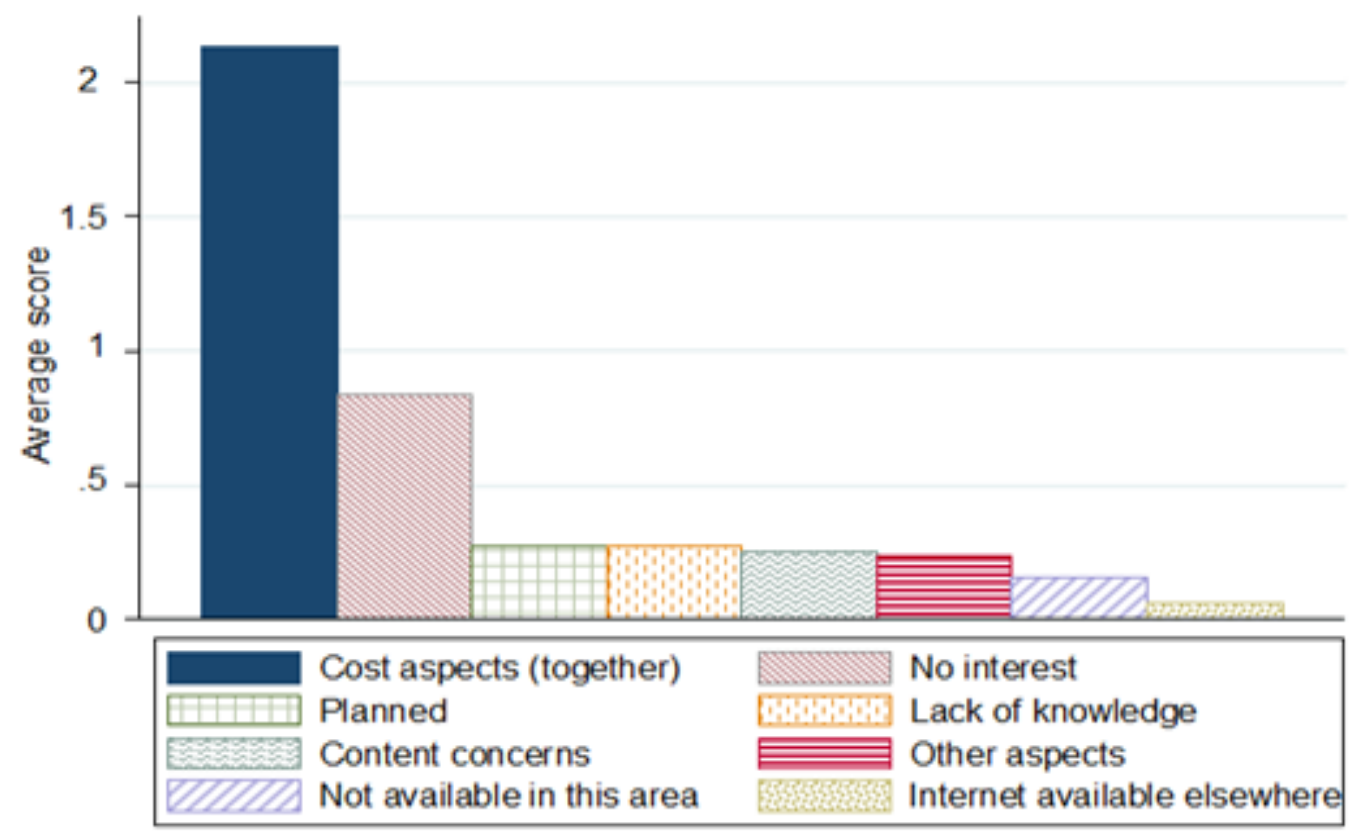


Figure 4

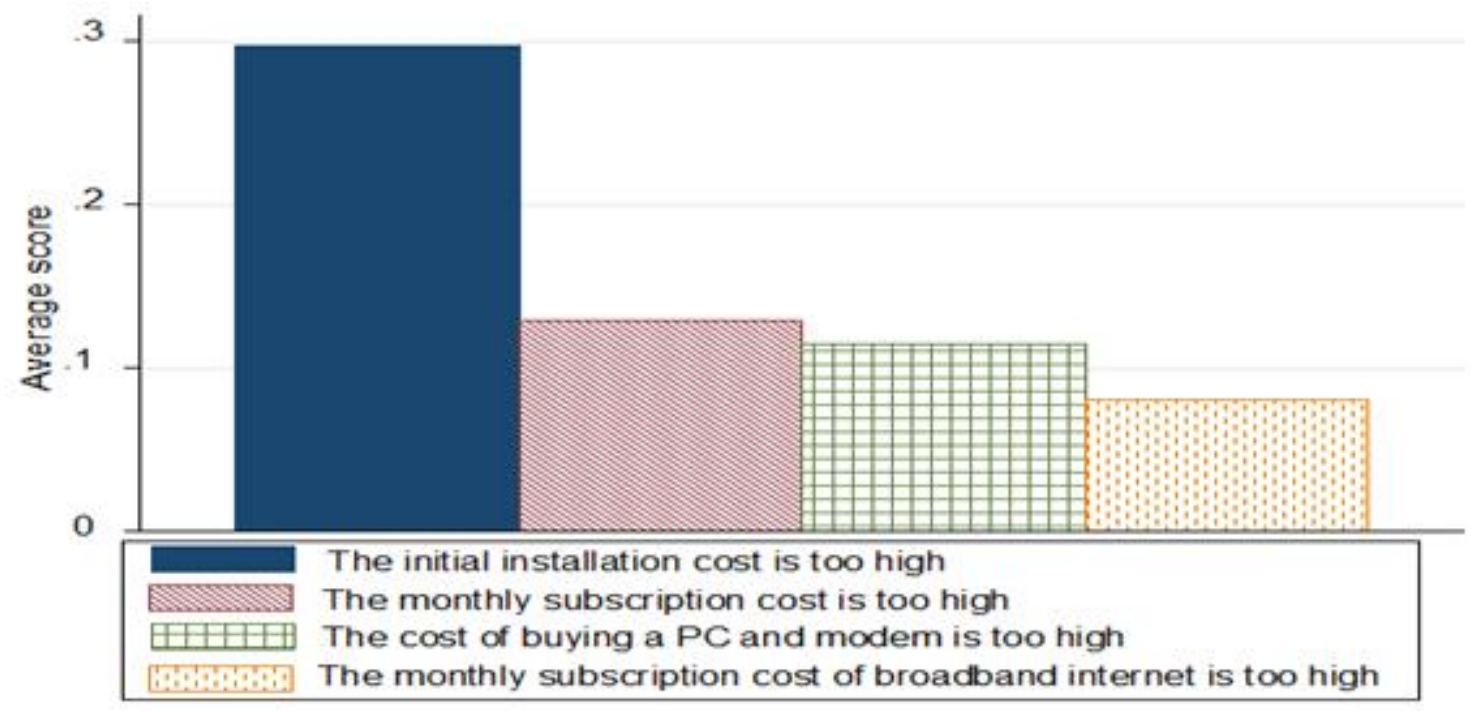

